\title{
Do You Need the WET: A Modest Proposal
}

\author{
S. J. Kleinman
}

Gemini Observatory, 670 N. A'ohoku PI., Hilo HI USA 96720

The Whole Earth Telescope (WET) is an extremely powerful, but also extremely resource-demanding, tool. To help ensure the WET's enormous resources are called into play only when they are truly needed, I urge potential users to seriously consider other options to getting the necessary data before turning to the WET and I urge the WET to consider ways to establish a mechanism for obtaining large sets of single-site data for potential WET targets.

I want to raise one question which should be forefront in everyone's mind before an object is proposed or accepted as a WET target. Does your object really need extended coverage? The WET has become so well-established, so easy to use, that we often forget the immense amount of resources in time, money, and telescope access that a WET run costs. It is relatively easy to propose and observe a target with the WET these days. We've learned a lot since the early days and the WET runs very efficiently thanks to the dedicated hard work of the people at headquarters and elsewhere. It can seem quite simple, appropriate, and even logical to use the WET simply as a mechanism for obtaining lots of data on a target, and not necessarily as one only to obtain lots of extended coverage data on an object. Compounded by a growing list of new variables and new WET collaborators and science ideas, this alreadyexisting temptation becomes even more pronounced.

What makes the WET unique are the quality and dedication of its people and its concentration on extended, 'round-the-globe coverage of its target stars. The utilization of these great strengths requires an immense amount of resource expenditure and we do a dis-service to the WET and its members when we call the WET into being for an observation that does not absolutely require its unique strengths and resources.

The WET enjoyed a large amount of very early success. The main reason being that the WET was born out of necessity. There was already a pre-existing backlog of extensive, yet unexplained, single-site data that we knew had to be supplemented by extended coverage data if we were to ever understand them. The existing single-site data nearly guaranteed that breaking the 1-day alias in 
our data would produce scientific discoveries. We knew both that the WET observations were going to give us new information that we had no other way of obtaining and that this information would lead us directly to new results.

Thanks to the large increase in the number of known white dwarf and other pulsators from the Sloan Digital Sky Survey (eg.Mukadam et al. 2004 and Nitta et al. 2005) and other efforts, we have a lot of new potentially-interesting targets to observe. However, being new discoveries, many of these objects lack significant single-, let alone multi-site data that justify the necessity of the WET. In addition, many of the new variables are $\operatorname{dim}(g \approx 19,20 \mathrm{mag})$ and require $2-4 m$ class telescopes to observe. This means there is more competition for telescope time than with $1-2 \mathrm{~m}$ class objects and even more requires us to have solid background data to justify the use of a very large percentage of the world's 2-4m telescopes for a continuous 2-week period.

We therefore need to concentrate on using the WET only when its strengths are fully justified. To this end, we need to start gathering background single/multisite data on these new variables to determine their suitability for the WET. With the WET taking up a couple weeks of telescope time a year on many of the suitable telescopes/instruments for our work, there aren't many options left to getting additional time for this background work.

One solution would be to obtain access to a dedicated, remote-operated, suitable telescope, perhaps something like the Las Cumbres network ${ }^{1}$. The WET provides ample opportunity for shared learning, education, and public relations, so the match up should be fairly easy to sell. Alternatively, or maybe in addition, instead of a single WET run concentrating on one or two objects, we could do a non-WET run using similar resources, but gathering data on many different objects during the run. We could even consider adjusting which telescope observes which objects in an effort to optimally affect alias patterns.

These are just two possible ideas to obtain necessary pre-WET data. There are certainly others, but what is clear to me, is that in the end, we must be able to prove before the WET is ever turned to an object that unique results are likely to follow. The WET is too costly a tool to employ when it is not absolutely necessary. We must always ask and answer the question: Does this object really need the WET?

\section{References}

Mukadam, A., Mullally, F., Nather, R. E., et al. 2004, ApJ, 607, 982

Nitta A., Kleinman, S. J., Krzesinski, J., et al. 2005, ASPC, 334, 585

\footnotetext{
${ }^{1}$ http://lcogt.net/
} 


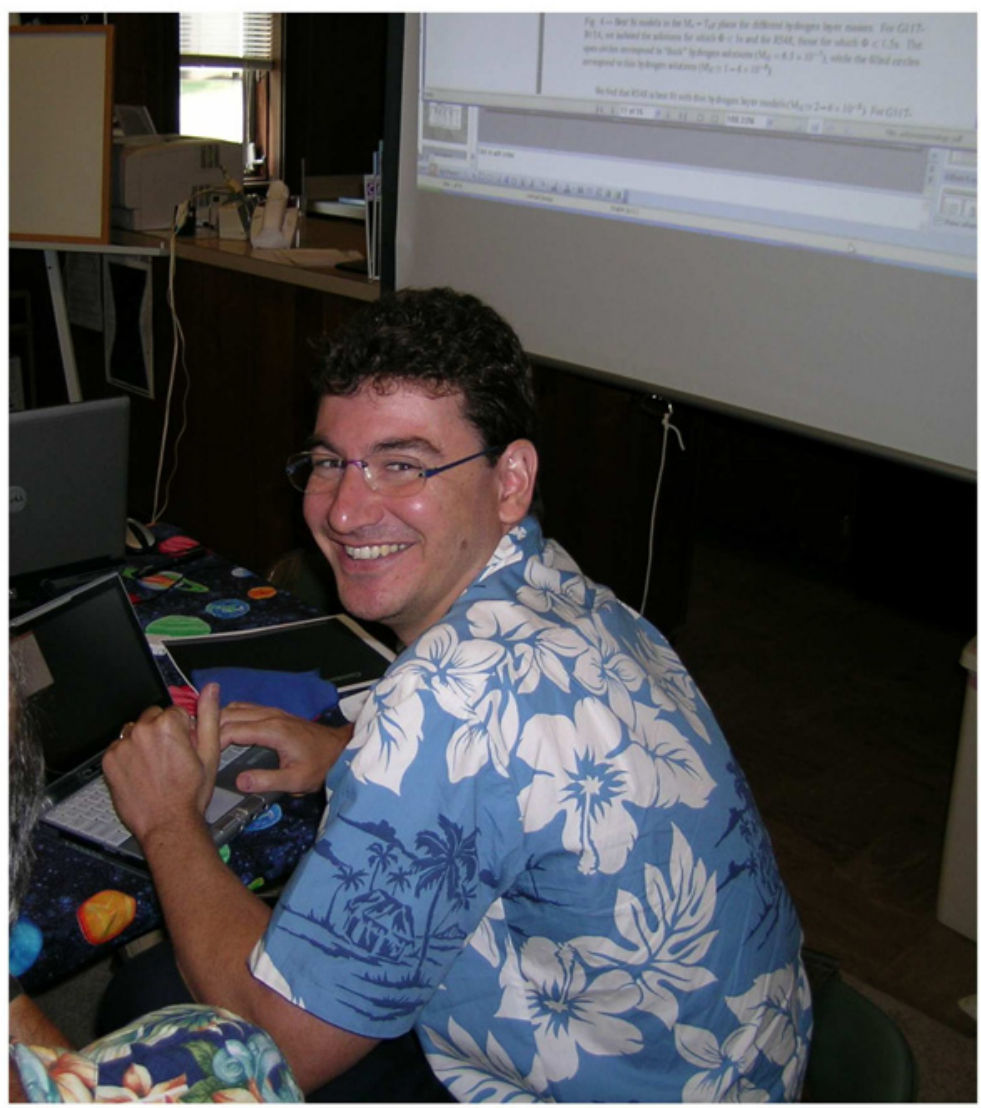

S. Kleinman enjoying the afternoon talks. 